\section{Gestión del Turismo como Integrador \\ de la Iniciativa Internacional y Regional \\ en el Actual Proceso Selectivo de la Globalización. Estudio de caso Sierra de la Capibara - Brasil \\ Tourism Management as an Integrator of International and Regional Initiative in the Current Selective Process of Globalization. Case Study Sierra de la Capibara - Brazil}

Geraldo Luciano Toledo Eliana Marroquin Quelopana ${ }^{2}$ Álvaro Castroman Pollero ${ }^{3}$

RESUMO: En una región de América Latina con bajos índices de desarrollo humano - IDH, se quiere implementar un modelo turístico que permita la integración de la región potencialmente turística con los beneficios del turismo internacional. La opción del turismo se debe a que la región de la Sierra de la Capibara, Brasil, posee un recurso declarado Patrimonio Cultural de la Humanidad, y se intenta poner en valor dicho recurso turístico, que ya cuenta con la inversión de un grupo internacional en un resort de 400 millones de dólares.

1. Licenciado y doctor en Administración por la Facultad de Economía, Administración y Contabilidad de la Universidad de São Paulo - FEA-USP; vice-director (1994-1998) y profesor titular del Departamento de Administración de la FEA-USP; autor de libros y diversos artículos publicados en las áreas de marketing estratégico y de servicios, planificación estratégica y administración estadística. Contacto: Av. Prof. Luciano Gualberto, 908 sala E - 106, FEA, Cidade Universitária - 005508-900 - São Paulo-sp; e-mail: e-mail: gltoledo@usp.br.

2. Doctoranda en la Escuela Técnica Superior de la Universidad Politécnica de Madrid; master stricto sensu en Administración de Empresas por la Universidad de São Paulo; ingeniera en Informática por la Universidad Femenina del Sagrado Corazón, Lima; consultora en gestión del conocimiento e innovación en empresas públicas y privadas en Perú, Brasil y España. Contacto: Mar Caspio 49 - 28033 - Madrid, España; e-mail: eliana_marroquin@yahoo.es.

3. Doctorando en Turismo en la Universidad de São Paulo - USP; mestre en Integración de América Latina por la Usp; profesor de la Universidad del Uruguay - UTU; planificador de las estrategias técnicas-económicas del órgano estatal de Punta del Este, Uruguay; técnico integrante da comisión mixta de turismo Uruguay-Argentina - CMT. Contacto: Av. Prof. Luciano Gualberto, 908, sala E - 106, FEA, Cidade Universitária - 005508-900 - São Paulo-sp; e - mail: alvarocp@usp.br.
El objetivo de este estudio es determinar la posibilidad que el proyecto permita la integración de la iniciativa privada, pública y regional para estructurar el desarrollo de esa región. La metodología es un enfoque territorial multicriterios de naturaleza selectiva y estratégica. Los resultados apuntan la complejidad socioterritorial y económica de la integración de los diferentes agentes para conseguir el desarrollo turístico sostenible.

PALABRAS CLAVE: turismo; desarrollo turístico regional sostenible; iniciativa privada y regional.

ABSTRACT: In a region of Latin America, with lower indicators IDH, is tried to implement a tourist model that allows the integration of the tourist region with the benefits of the international tourism. The option of the tourism is due to that the Sierra de la Capibara - Brazil possesses a resource declared Cultural Patrimony of Humanity; it tries to put on value this resource to lead the project that consists with the investment of an international group in a resort of 400 million dollars. The objective of this study is to evaluate the possibility that the project allows the integration of the private, public and regional initiative to structure the tourist regional development. The results stand out the partner-territorial and economic complexity of the integration of the different agents to reach tourist sustainable development.

KEYWORDS: tourism; regional development of sustainable tourism; private and regional initiative.

\section{Introducción}

Todas las regiones están impactadas por la actual fase de la globalización; sin embargo, hay pocas beneficiadas. La globalización, por ser un proceso selectivo, crea un proceso dinámico y de alta complejidad, práctica-territorial, en poder implementar las teorías de desarrollo sostenible en regiones deprimidas (Molina, 2005).

Como integrar las regiones con recursos turísticos de América Latina con los mercados emisores, como Estados Unidos y Europa, es un desafío que trasciende el marco regional y necesita de la cooperación de diferentes agentes como: inversores privados, instituciones públicas, ongs y la participación de la población local, dentro de una plataforma política técnico-comercial que permita determinar los objetivos comunes y el marco regulatorio en que se desarrollara el turismo regional.

La competitividad del turismo mundial ha evolucionado de tal manera que exige cada vez más la contemplación de las especificidades regionales. La valorización de las particularidades ha permitido que antiguas regiones ignoradas por el turismo internacional ahora puedan ser incluidas. En el caso de regiones 
deprimidas y con recursos turísticos, dicha inclusión al sistema internacional del turismo puede traer un conjunto de beneficios y/o inconvenientes intrínsicos de la actual etapa de la globalización.

Para optimizar los beneficios del turismo dentro del desarrollo regional se deben considerar entre otros aspectos su tejido social, institucional y cultural; su marco político; su medio ambiente; su sistema productivo; sus distintas lógicas de funcionamiento y estructura regional. Esa complejidad multicausal e imbricada obliga a evitar simplificaciones y reduccionismos - principalmente el economicista - sobre todo en las sociedades menos desarrolladas. Las regiones deben pasar a ser agentes activos en la globalización, dejando de ser simples "campos de maniobras" para ser "actores" decisivos de su desarrollo.

En este trabajo se estudia la región de la Sierra de la Capibara, Brasil, declarada, en el año 1992, Patrimonio Cultural de la Humanidad por la uNEsCo (2004). Posee más de setecientos sitios arqueológicos, que datan entre 20.000 y 50.000 años atrás. Es una región con bajos Índices de Desarrollo Humano - IDH (PNUD, 2004). Esta deprimida situación regional llevó al gobierno brasileño a estudiar la posibilidad de implementar un modelo de desarrollo sostenible articulado por el turismo como actividad transversal-integradora del desarrollo regional.

El turismo es escogido como el articulador del desarrollo de esa región principalmente por dos motivos: primero, por el volumen de recursos turísticos que la región posee, que la hacen una singularidad histórica-cultural única en el mundo; segundo, porque la región no posee muchas otras posibilidades socioeconómicas que puedan mover el desarrollo sostenible, como podrían ser la agropecuaria, minería, comercio, etc.

Como hito regional, existe a partir de la futura inauguración del aeropuerto intercontinental, en el año 2007, la posibilidad de un punto de inflexión en la región para el desarrollo sostenible. Al terminarse de construir el aeropuerto el flujo actual de turista saltará de 5 mil al año a 1 millón al año (PIEMTUR, 2003), lo que posibilitaría el desarrollo del turismo de masa, que presentaría la entrada a un alto volumen de inversiones (directas e indirectas) económicas e institucionales, como la construcción del resort cinco estrellas. Así la inauguración del aeropuerto desataría un proceso en cascada de inversiones económicas públicas-privadas e institucionales en la región. Aunque la esfera de las inversiones económicas esté confirmada, por el contrario todavía no están correlacionadas con acciones preventivas o programas de desarrollo social e institucional para la comunidad local.

Analizando la teoría de desarrollo sostenible, ese olvido de la esfera social, cultual e institucional de la regional no conduciría a dicho desarrollo. Como diferencian Rodríguez (2004) y Molina (2005), desarrollo sostenible es un concepto con mayor alcance que el crecimiento económico, pues el crecimiento se refiere al aumento relativo del Producto Interior Bruto - PIB Y, en consecuencia, es solo un elemento del desarrollo sostenible. Las inversiones, del aeropuerto y del resort, sin dudas, aumentarán el PІв regional, pero conseguir el desarrollo sostenible es más ambicioso, y por lo tanto más complejo, arduo y dificultoso que conseguir el crecimiento económico.

Por lo tanto en este estudio se intenta saber como el turismo, en la actual fase de la globalización, puede - o no - configurar el desarrollo regional en regiones deprimidas de América Latina:

1. Especialmente en una región con los índices más bajos de IDH $(0,677)$ ¿Existe la posibilidad de implementar un desarrollo de manera sostenible que incluya la mayoría de la población local?

2. La región posee un patrimonio cultural de la humanidad. ¿Puede ese patrimonio ser el motor de un desarrollo a partir del turismo como la actividad coordinadora de ese mismo desarrollo?

3. Con el aval del gobierno central de Brasil se construirá un resort turístico de 400 millones de dólares en la región. El agente es internacional, agrupa a quinientos tour operadores de todo el mundo. Sin dudas, traerá un crecimiento económico para una región deprimida como la Sierra de la Capibara. ¿Traerá también un desarrollo sostenible?

Así, el objeto de estudio es la actual configuración de la región dentro del posible proyecto de desarrollo, con especial cuidado para los impactos o sinergias del turismo de masa. El objetivo es determinar la posibilidad que el proyecto permita la integración de la iniciativa privada, política y social de la región para estructurar el desarrollo sostenible regional turístico.

La metodología es un enfoque territorial multicriterios, estratégica y selectiva. Se aplica los métodos de UCM (2004) у омт (2005). Los resultados apuntan de la complejidad socioterritorial y económica de la integración de los diferentes agentes para conseguir el desarrollo turístico.

\section{Revisión de la literatura}

\section{Globalización y territorio}

Molina (2005) describe que la complejidad de la globalización, sin duda es el proceso que más influye en la dinámica de la sociedad actual, beneficiosa para algunos territorios ó destructiva para otros. En este estudio, se analiza la globa- 
lización desde la perspectiva del desarrollo regional en lugares no integrados a los beneficios de dicho proceso. La dinámica de globalización genera nuevas e importantes desigualdades sociales y territoriales, afectando más adversamente a los más pobres. Para Molina (2005) el cuestionamiento de lo global y local pasa, al contrario de lo que algunos autores predecían, por la revalorización del papel del territorio y de sus recursos específicos en la actual fase de la globalización.

Por lo tanto el análisis de lo global y local pasa a ser primordial en esta investigación. Se tiene como referencia los conceptos planteados por Santos (1996) de las "horizontalidades" y "verticalidades", como se esquematiza en la Figura 1; donde se debe considerar la multicausalidad imbricada en el tiempo-espacio de los flujos verticales (que son guiados por procesos como: globalización, competitividad, sistemas de producción entre otros) con los flujos horizontales (que son construidos por acciones de planificación, gestión, solidaridad y cooperación regional; por el aumento del capital social; por el aumento del desarrollo del tejido cultural, social y político; por el aumento de la calidad de vida de la población local; entre otros).

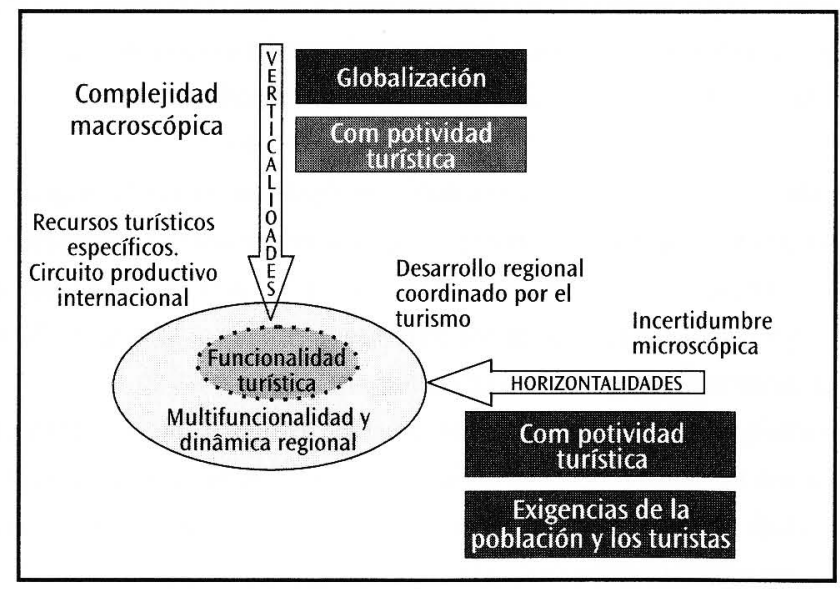

Fontes: Autores

Figura 1. Horizontalidades y verticalidades de una región turística. Flujo interpretativo de la funcionalidad turística

Las características de la globalización llevan a que los actuales sistemas económicos y territoriales se caractericen por su intrínseca complejidad (Camagni, 2003). Surge así, una rica y multiforme teoría evolutiva del desarrollo territorial, que se vincula paralelamente a una trayectoria semejante al pensamiento de otros ámbitos, como los de: economía, ciencias empresariales, progreso técnico-innova- ción, entre otras. Por lo tanto la dinámica territorial se convierte en un proceso de investigación en evolución, que es un programa científico distinto del pasado, ya que ahora se trata de interpretar el cambio estructural, la innovación territorial y la casualidad para poder entender la dinámica territorial (Camagni, 1998).

Para Molina (2005) el desarrollo local debe estar unido al impulso y consolidación de nuevas actividades. Cita algunas de las estrategias del desarrollo local:

- operar sobre la base territorio municipal y actuar sobre un territorio amplio y, a ser posible, cohesionado social y políticamente;

- optimizar la calidad de los servicios y en especial las inversiones;

- espacio jerarquizado en las necesidades pero sin la exclusión de nadie;

- inversiones importantes en infraestructura, en servicios y en formación de capital humanos. Pacto político-social lo más amplio posible;

- carácter integrador y participativo social. Mesas territoriales plurales, etc. Frente al individualismo, trabajar en conjunto en los diversos sectores y escalas. Superar el victimismo y acometer acciones emprendedoras;

- determinar objetivos y dimensiones estratégicas. Aprovechamiento sostenible de los recursos;

- otros.

\section{Recursos regionales específicos y globalización}

Méndez (2003) considera que para mejorar la identificación de los recursos potenciales para el desarrollo de un territorio, en la actual fase de la globalización, los recursos pueden ser estructurados sobre la distinción entre genéricos y específicos. Para el autor los recursos específicos son: recursos humanos, estructura empresarial, infraestructuras (centros de I+D, laboratorios, etc.), recursos patrimoniales (natural, paisaje, histórico, cultural), entorno sociocultural (valores culturales compartidos, participación-capacidad de auto organización, confianzaconcertación, solidaridad y ayuda mutua).

Vázquez Barquero (2002) afirma que las teorías de desarrollo en esta fase de la globalización pasan a considerar al territorio como un agente de transformación sobrepasando la consideración antigua de ser solo un suporte para los recursos y actividades. Los recursos específicos pasan a ser una compleja configuración dinámica e integrada de recursos construidos socialmente. Son difíciles de imitar o sustituir y deben ser incluidos dentro del proyecto futuro de la región.

Para Molina (2005), el objetivo de desarrollo local es basado en las singularidades locales, o sea a partir de estos recursos específicos está una de las pocas salidas, en la actualidad, a la crisis de las viejas funciones socioeconómicas y a la 
emergencia de otras nuevas, aprovechando las oportunidades y previniéndose de las amenazas del proceso de globalización.

En la dinámica, expuesta por Molina (2005), se debe saber cual es el recurso estratégico, se debe proseguir con su valoración para posteriormente darle un precio. La valorización de los recursos específicos dentro de un modelo de desarrollo, en la actual globalización, debe tener una filosofía de mayor alcance que los modelos económicos, sabiendo, como explica Santos (2000), quienes deben ser sus reales beneficiarios prioritarios dentro de esos modelos: la mayoría de la población local.

\section{Un recurso específico en las regiones deprimidas: actividad turística}

Los cambios socioeconómicos y territoriales de la última década están haciendo emerger, aun cuando todavía sea de forma tímida, nuevos factores de desarrollo. El turismo ha sobrepasado los padrones tradicionales de sol y playa o de pistas de nieve y ha comenzado ha valorizar territorios a partir de diferentes recursos medioambientales, del patrimonio sociocultural, del paisaje, historia, tradiciones, etc. (Troitiño et al., 2004)

Así el turismo, en la actual fase de la globalización, es considerado como una de las más recurridas estrategias activas de puesta en valor de los recursos territoriales de las regiones menos desarrolladas. A su favor son autores como Veltz (2001), Santos (2000), Vázquez Barquero (2002), Méndez (2003), Troitiño et al., (2004), Molina (2005), entre otros.

El turismo puede ser la actividad valorizadora de los recursos regionales en recursos específicos en la globalización, una vez que, como afirman Toledo et al. (2004), posee características de motor regional en regiones deprimidas económicamente principalmente por su:

- Multisectorialidad. La EMBRATUR (2003) afirma que en las regiones turísticas de Brasil el turismo afecta de manera directa o indirecta más de 52 sectores de la economía regional;

- Multifuncionalidad. El turismo puede revitalizar las antiguas actividades y/o crear nuevas actividades socioeconómicas que ayuden al desarrollo regional (Santos, 2000);

- Sostenibilidad. El turismo adecuadamente planificado, implementado y gestionado puede ser una actividad sostenible en nivel regional;

- Transversalidad-integradora. La adecuada gestión de las características anteriores le dan la capacidad al turismo de ser la actividad que lidere e integre transversalmente los demás sectores de la región (Troitiño, 2003).
En diferentes regiones de América Latina, la actividad turística ha despertado un gran abanico de expectativas sobre los resultados de su actuación, muchas veces ilusorias y mal dimensionadas técnicamente. Las expectativas se reflejan en diferentes y complejos intereses, no siempre alcanzados, en todas las esferas de la sociedad. En regiones deprimidas, se sabe lo complejo, dinámico y arduo que es planificar, transversalmente e y de manera integrada, el turismo para que la sociedad local sea la mayor beneficiaria. Toledo et al. (2004) al estudiar el desarrollo sostenible en regiones latinoamericanas turísticas, concluyen que existen regiones en las siguientes etapas de desarrollo turístico: inicio, desarrollo, consolidación y declive. Notase que la región de estudio no entraría en ninguna de estas clasificaciones, ya que a pesar de poseer un rico recurso turístico, su ciclo turístico está lejos de ser calificado de inicio.

\section{Proyectos turisticos en regiones deprimidas}

La implementación de un proyecto turístico en una región deprimida no es directa ni lineal. Existen diversos obstáculos y dispersiones en la etapa de implementación, aunque su elaboración haya sido excelente. Como afirma Rodríguez (2004), la mayoría de los proyectos fallan al considerar que el espacio es un contenedor neutro, mientras que la apariencia y forma del espacio vienen determinadas por como se han conjugado los diferentes procesos de transformación en el pasado y por la realidad presente. En el espacio confluyen las diferentes fuerzas que constituyen la sociedad; se enfrentan los distintos ritmos de transformación de las esferas sociales; y colisionan innovación con tradición. El espacio turístico solo puede ser comprendido teniendo en cuenta los procesos multicausales e imbricados de sus fijos y flujos.

Para Rodríguez (2004), en cuanto una empresa internacional se instala en un territorio menos desarrollado, su actividad económica es de una efectividad territorial casi inmediata y lineal, mientras que la esfera cultural, política y social es mucho más tardía y compleja.

La actividad turística, para poder ser el líder transversal-integradora del desarrollo regional, primero debe ser comprendida como una actividad más, dentro de la dinámica multifuncional más compleja de su región (Troitiño, 2003). Posteriormente es que debe ser valorizada con un rico y dinámico proceso de planificación, gestión, promoción y comercialización en que se deben incluir una serie de agentes públicos, políticos, empresariales, sociales, culturales y económicos. 


\section{Turismo como recurso específico para el desarrollo sostenible}

El concepto de desarrollo sostenible es cada vez más utilizado y exigido por los organismos sociopolíticos y técnicos dentro de sus exigencias técnicas y legales, entre ellos son: gobierno central, ministerios, entidades financieras, organismos multilaterais (BID, BIRD, etc.), entre otras. El concepto sostenible también es un hito que supuestamente guía teóricamente la actuación y directrices de los municipios, ONGS y diferentes entidades de las regiones latinoamericanas y de todo el mundo

Existe un rico y sólido conjunto de teorías científicas sobre la sostenibilidade. Ese marco teórico posee la capacidad de continuo mejoramiento teórico y actualización con los resultados de casos prácticos ejecutados en diferentes regiones del mundo. En esta investigación hay conciencia de las limitaciones y peligros del uso del término sostenibilidad y sostenible, por eso siempre asume que para utilizar esos conceptos se debe incluir todo su contenido técnico-metodológico, aunque en la mayoría de las veces su uso queda solo en la cáscara y no entra en el contenido llegando al extremo de engaño o de la mala intención de su uso. En el Cuadro 1 se relaciona la evolución institucional-política del marco técnico de la sostenibilidad.

Cuadro 1: Eventos importantes sobre desarrollo sostenible y turismo

\begin{tabular}{|l|l|}
\hline Ano & \multicolumn{1}{|c|}{ Evento } \\
\hline 1972 & Conferencia de la onu de Medio Humano, Estocolmo. \\
\hline 1972 & Publicación informe del Club de Roma: los límites del crecimiento. \\
\hline 1982 & Publicación informe Global 2000. \\
\hline 1984 & Responsible Care (Compromiso de Progreso). Autorregulación química. \\
\hline 1987 & Publicación informe Nuestro Porvenir Común - cmMAD (Informe Brutland). \\
\hline 1991 & Cámara Comercial Internacional. Carta Empresas: Desarrollo Sostenible. \\
\hline 1991 & Publicación informe Club de Roma: además de los límites del crecimiento. \\
\hline 1992 & Conferencia de la onu del Medio Ambiente y Desarrollo. Agenda 21, Rio de Janeiro. \\
\hline 1995 & Informe onu Reunión Mundial Desarrollo Social, Copenhague. \\
\hline 1995 & Carta del Turismo Sostenible, Lanzarote - España. \\
\hline 2000 & Ciudades Sustentables. Sostenibilidad local. Indicadores Comunes Europeos. UE. \\
\hline 2001 & Código Ético Mundial para el Turismo. onu. \\
\hline 2000 & Informe onu Declaración do Milenio de New York. \\
\hline 2002 & Informe onu Conferencia Mundial: Desarrollo Sostenible, Johannesburgo. \\
\hline 2002 & Declaración de Québec: Ecoturismo - omt e Informe Desarrollo Humano - PNUD. \\
\hline
\end{tabular}

Fuente: Los autores.
Específicamente en el sector de turismo, a partir de la Conferencia ECo 92 se crea la Agenda 21 del Turismo. Así, comienza ha divulgarse y sistematizarse más integralmente los conceptos de sostenibilidad y turismo. La OMT (2005) define el desarrollo turístico sostenible en una determinada región como:

[...] aquel desarrollo que satisface las necesidades presentes y futuras de los turistas y de la región anfitriona conjuntamente protege y revaloriza las futuras oportunidades regionales. Esto significa prever como se conducirá la gestión de los recursos: económicos, sociales, culturales y ambientales de manera que puedan ser desarrollados manteniendo la integridad cultural, proceso ecológico esencial, diversidad biológica y el sistema de soporte de vida regional.

Para Méndez (2003), el desarrollo sostenible a partir del turismo:

Debe poseer la capacidad de generar e incorporar conocimientos para dar respuestas creativas a los problemas presente, (...) no solo en términos de crecimiento económico, sino desde una perspectiva más atenta a la calidad de vida que pueda disfrutar su población.

\section{Grado de efectividad de los procesos sociales en la actual globalización}

A partir de las teorías de Santos (1997) y Rodríguez (2004) se pretende desarrollar el concepto de efectividad, en el tiempo, de las diferentes actividades - sociales, económicas, culturales y políticas - en la implementación de un proyecto de desarrollo turístico en una región deprimida, como es la de este estudio.

La concepción de territorio, para Santos (1997), es la región más la esfera política, o sea, es un mosaico de elementos de diferentes eras que sintetiza, de un lado, la evolución de la sociedad, y explica, de otro lado, situaciones que se presentan en la actualidad. Actualmente, la esfera económica globalizada posee una mayor efectividad territorial que las esferas social, cultural y política. Esas diferentes velocidades de las efectividades en el territorio, para Rodríguez (2004), es debido al peso de la herencia cultual, las diferencias políticas, la ausencia de órganos de legislación supranacionales eficaces en asuntos sociales, entre otros factores, y son los que inducen a que la esfera social, cultural y política esté mucho más circunscripta a la escala regional y local que la propia esfera económica.

Rodríguez (2004) concluye que la efectividad de la dimensión política, social y cultural sigue siendo determinada, en un alto porcentaje, por las particularidades regionales en cuanto la efectividad económica no. $\mathrm{O}$ sea en el actual proceso de globalización y en regiones deprimidas, como se muestra en el Cuadro 2, las primeras efectividades en manifestarse en los territorios son los cambios econó- 
micos. Posteriormente se manifiestan las transformaciones políticas, sociales y culturales, aunque, si es que se dan, solo serán a largo plazo. Por eso hay autores que afirman que en América Latina se viven realidades paralelas, superpuestas con aceleraciones y acceso a los beneficios de la globalización diferentes, dentro de un mismo territorio.

Cuadro 2. La efectividad de los procesos de la globalización en una región deprimida

\begin{tabular}{|l|l|l|l|l|}
\hline Plazo/Esfera & Económica & Social & Política & Cultural \\
\hline Corto Plazo & & & & \\
\hline Medio Plazo & & & & \\
\hline Largo Plazo & & & & \\
\hline
\end{tabular}

Fuente: Rodríguez (2004).

Analizando el Cuadro 2, Rodríguez (2004) lo explica:

[...] la estructura y funcionalidad espacial heredada se convierte, entonces, en una condicionante que a la larga va a conferir a unos procesos de transformación diferentes velocidades de efectividad dentro del territorio.

\section{Metodología}

La perspectiva adoptada, en el estudio, responde a un enfoque territorial multicriterios, de naturaleza estratégica y selectiva, orientado desde el primer momento a identificar problemáticas, dinámicas y tipologías tanto de la configuración regional como las oportunidades para implementar un proyecto de desarrollo regional a partir del turismo.

Se basa así, en Troitiño et al. (2004), que señalan que para una investigación de campo es aconsejable adoptar una metodología integradora y relacional, de base geográfica apoyada en indagaciones sucesivas, a efectos de identificar de forma precisa los recursos de dimensiones múltiples que posee el territorio, la comprensión relacional-territorial, y reflejar las interdependencias entre los factores ambientales, económicos, políticos, sociales y culturales.

En este trabajo, la interpretación de los resultados se hace a partir de las consideraciones y encadenamiento lógico, de la revisión de la literatura en temas como globalización, territorio, desarrollo, sostenibilidad, recursos específicos y fases de efectividad de las distintas esferas. El enfoque del trabajo de campo se realizó de acuerdo a la valorización de los siguientes multicriterios (Troitiño, 1998) temáticos de diagnóstico y del análisis detallado por:

1. Patrimonios histórico e cultural,

2. Infraestructura y equipamientos para el turismo,

3. Flujos turísticos y capacidad de acogida turística,

4. Gestión comercial del turismo,

5. Impactos económicos y funcionales,

6. Impactos socioculturales,

7. Accesibilidad y movilidad turística,

8. Turismo, urbanismo y medio ambiente urbano,

9. Políticas turísticas, instrumentos de planificación e infraestructuras de gestión.

\section{Fases metodológicas}

Fue utilizada como técnica de recogida de datos el procesamiento en fuentes secundarias, que comprende los referenciales bibliográficos, artículos y otros documentos e investigaciones sobre el tema en estudio. Más adelante, fue efectuado un procesamiento de la información recogida en la región de la Sierra de la Capibara, Brasil. Estas informaciones fueron generadas de fuentes primarias mediante: reuniones pre-planificadas; entrevista semi-estructurada individual, a través de un cuestionario con preguntas directas a técnicos; cuestionarios a la población local; inventarios, e-observación directa. Las reuniones técnicas fueron realizadas en diferentes sectores, instituciones y organizaciones privadas y no gubernamentales, realizando más de treinta reuniones con los líderes regionales, donde se aplicaron técnicas como: reuniones técnicas participativas-dinámicas; entrevistas semi-estructuradas individuales; inventarios, topologías y levantamientos de datos técnicos; observación directa.

En la visita técnica se aplicó la metodología de la UCM (2004) y de la OMT (2005) que permiten recoger un conjunto relevante de informaciones durante el trabajo de campo. La interpretación de estas informaciones tuvieron como resultado la caracterización y formulación de la problemática que identificó las posibilidades de la configuración regional para implementar un proyecto de desarrollo, donde el turismo sea la actividad transversal-integradora de desarrollo sostenible regional. 


\section{Región de estudio}

La Sierra de la Capibara está situada en el sur este del Estado de Piauí, en la región Nordeste de Brasil. Ha sido declarado Parque Nacional en 1979 por poseer la mayor concentración de sitios arqueológicos prehistóricos de América. Existen más de setecientos sitios que presentan los vestigios más antiguos de la presencia humana en toda América. Se remontan entre 20.000 y 50.000 años, según la FUNDHAM (2003). Posee un área de 130 mil hectáreas y su gestión y protección quedó bajo el ıвAмA y la legislación brasileña de parques nacionales. En 1992, por la importancia de sus sitios arqueológicos, la UNESCO (2004) registra al parque dentro de la lista de Patrimonio Cultural de la Humanidad.

\section{Investigación de campo}

A manera de síntesis, la Tabla 1 presenta algunas de las características más relevantes para la esfera del turismo que fueron constatadas pro la aplicación de la metodología de la UСм (2004) у омт (2005).

Tabla 1: Debilidades y oportunidades más relevantes para la actividad turística

Recursos e infraestruturas culturales

\begin{tabular}{|l|}
\hline \multicolumn{1}{|c|}{ Recursos e infraestruturas culturales } \\
\hline - Figuras prehistóricas con altísimo potencial de atracción turística en ámbito mundial y es un \\
atractivo con potencial de visita de 2 millones turistas/año. Museo con potencialidades de \\
desarrollo. \\
- Existe potencial para turismos secundarios como son: ecoturismo y turismo de aventura. \\
- Falta técnica de diagnóstico, desarrollo, gestión y articulación de los atractivos turísticos \\
secundarios. \\
Flujos turístico, infraestructuras e incidencias económicas y funcionalidades \\
\hline - Todo esta muy incipiente. Existe a partir de la construcción del aeropuerto la posibilidad de \\
un punto de inflexión en la región. Al construirse el aeropuerto el flujo de turista de 5 mil \\
al año saltaría a 1 millón (PIEMTur, 2003) y se construiría un resort cinco estrellas con capital \\
italiano (400 millones de dólares) y para 30 mil camas en cuatro años. \\
- Precariedad de los equipamientos hoteleros, restauración y demás infraestructuras turísticas. \\
Servicios turísticos son de baja capacitación. \\
- Falta de agua potable, energía, saneamiento, tratamiento de residuos y comunicaciones. \\
- El turismo tiene incidencia muy baja en la población local.
\end{tabular}

- La funcionalidad de la región es la agropecuaria de subsistencia, cabruno cultura, funcionarios públicos, comercio ciudadano y planos de ayuda.

- No hay otras actividades socioeconómicas con perspectiva de mejorar la región.

$$
\text { Accesibilidad, movilidad y medio ambiente }
$$

- Aislamiento regional. La accesibilidad sin aeropuerto no tiene posibilidades de mejorar

- El parque tiene buena movilidad para 5 mil excursionistas. La movilidad parque-ciudades es muy precaria. Movilidad entre otros recursos turísticos regionales también es precaria.

- La movilidad de la ciudad es muy precaria. Su trazado viario y sistema comercial son precarios.

- Mala cohesión social en las ciudades y ciudades-campo.

- Medio ambiente: problemas de residuos sólidos, aguas pluviales y saneamiento.

$$
\text { Infraestructuras y gestión comercial del turismo }
$$

- Inexistente red de comercialización del producto turístico. Solo existe promoción instantánea y tres agencias pequeñas especializadas en turismo arqueológico.

- Falta de capacitación de los recursos humanos en cualquiera actividad turística.

- Cultura regional de la desconfianza y del individualismo.

- Población e instituciones locales favorables a la actividad turística.

Fuente: Elaboración propia.

\section{Estrategias para el desarrollo sostenible}

De la metodología de la UCM (2004) y омт (2005) fueron realizadas en las siguientes etapas: I: Análisis de la realidad; II: Diagnóstico e identificación de características más relevantes; in: Estrategias y lineas de actuación. Esas etapas permitieron obtener un rico conjunto de resultados, que se presenta una pequeña síntesis a seguir.

\section{Hito para el desarrollo sostenible del turismo}

La transformación del campo de aterrizaje de la ciudad de São Raimundo Nonato en aeropuerto internacional, con capacidad para todos los tipos de aviones, es el hito que debidamente aprovechado puede comenzar a estructurar el desarrollo sostenible del turismo regional.

Ese aeropuerto, desde su construcción, desencadenará una serie de actividades relacionadas al turismo. Aquí se describirán algunas de las actividades ya programadas al inicio de la primera fase del aeropuerto: 
Cuadro 3: Estrategias y acciones para la sostenibilidad

\begin{tabular}{|c|c|}
\hline Estrategia sobre: & Acciones o medidas sobre: \\
\hline $\begin{array}{l}\text { Patrimonio y oferta } \\
\text { complementaria }\end{array}$ & $\begin{array}{l}\text { Parque Nacional y el museo. } \\
\text { Desarrollo de artesanías, fiestas tradiciones regionales. } \\
\text { Articular circuitos turísticos regionales. } \\
\text { Inclusión en circuitos nacionales e internacionales. }\end{array}$ \\
\hline Alojamientos y restauración & Capacitar, mejorar y calificar alojamientos y restaurantes. \\
\hline Accesibilidad y movilidad & Estudios técnicos de la accesibilidad y movilidad. \\
\hline $\begin{array}{l}\text { Promoción y } \\
\text { comercialización }\end{array}$ & $\begin{array}{l}\text { Sinergia entre ILG, MINTUR, PIEMTUR, EMBRATUR. } \\
\text { Tour operador segmentados en el perfil del turista. } \\
\text { Tour operador que tienen circuitos en el Caribe y } \\
\text { América del Sur. }\end{array}$ \\
\hline $\begin{array}{l}\text { Información, formación y } \\
\text { sensibilización social }\end{array}$ & $\begin{array}{l}\text { Información técnica y social del ciclo turístico. } \\
\text { Formación y sensibilización social de desarrollo sostenible. } \\
\text { Plataforma gestora multinstitucional técnico-comercial para } \\
\text { la formación. }\end{array}$ \\
\hline $\begin{array}{l}\text { Flujos de visitantes y } \\
\text { capacidad de } \\
\text { acogida turística }\end{array}$ & $\begin{array}{l}\text { Programas de hospitalidad regional. } \\
\text { Programas conjuntos para turistas y población local. }\end{array}$ \\
\hline $\begin{array}{l}\text { Valorización del patrimonio } \\
\text { cultura y diseño de nuevos } \\
\text { productos }\end{array}$ & $\begin{array}{l}\text { Turismos: científico, ecológico, de aventura, tercera edad, de } \\
\text { estudiantes universitarios, pre wash (para aquellos que vie- } \\
\text { nen a realizar turismo de sol y playa y previamente realizan } \\
\text { un descanso en el parque) y otros. }\end{array}$ \\
\hline $\begin{array}{l}\text { Medio ambiente, } \\
\text { planificación urbana y } \\
\text { diversificación económica }\end{array}$ & $\begin{array}{l}\text { Fomento de negocios de la apicultura, certificación de los } \\
\text { viejos y nuevos guías turísticos, construcción de un restau- } \\
\text { rante en Dom Inocêncio y mejoría del taller de artesanía. } \\
\text { Capacitación y creación de una beneficiadora de leche de ca- } \\
\text { bra y producción de queso. Montaje y funcionamiento de la } \\
\text { posada escuela. Compra del horno eléctrico para el taller de } \\
\text { cerámica del parque. Inversiones en mejorías de los hoteles } \\
\text { existentes. Programas para dinamizar las nuevas actividades } \\
\text { socioeconómicas de la región y revitalizar aquellas activida- } \\
\text { des tradicionales como: agricultura, ganadería, etc. }\end{array}$ \\
\hline $\begin{array}{l}\text { Órganos e infraestructura } \\
\text { de gestión }\end{array}$ & $\begin{array}{l}\text { Institucional (Gobierno del Estado, PIEMTUR y Ministerio de } \\
\text { Turismo), FUnDHAM, Grupo ILG (de inversores, constructores y } \\
\text { gestores de los hoteles). }\end{array}$ \\
\hline Revisar el plano & $\begin{array}{l}\text { Planificación de preventiva transversal-integrada. } \\
\text { Beneficios directos a la población local. }\end{array}$ \\
\hline
\end{tabular}

Fuente: Elaboración propia.
También, a medida de síntesis, se enuncian algunos de los 157 programas y acciones que serán implementados en un plazo de tres años por una plataforma multiagentes en la región:

I - Programas

- Rescate de las tradiciones, artesanías y actividades culturales para ser incorporadas por la actividad turística;

- Programas educativos sobre el turismo. Medios: radio y programas de calle;

- Cursos de idiomas. Para guías, funcionarios de hoteles y sociedad local;

- Cursos de servicios de hotelería en la Posada Escuela;

- Certificaciones para actividades turísticas. Convenios con EMBraTur, etc;

- Cursos de capacitación de músicos y artistas regionales. SENAI, etc;

II - Estudios técnicos

- Impactos del turismo sobre el patrimonio natural y cultural.;

- Necesidades de infraestructuras básicas. (agua potable, transporte, comunicaciones, residuos sólidos y saneamiento, etc);

- Uso del suelo y plan de gestión del agua potable. Gestionar adecuadamente el uso del agua y evitar la especulación inmobiliaria a causa del turismo.

III - Ley Municipal para:

- programas escolares. Inclusión en la grade curricular de primero grado y segundo grado de disciplinas de Turismo y Medio Ambiente;

- capacitar los profesores.

IV - Planificación empresarial y comercial para:

- revitalización multisectorial y multifuncional con el turismo como actividad transversal-integradora;

- fomento de micro y pequeños negocios;

- preparación de la generación de empleos locales para la actividad turística;

- creación de negocios de restaurantes, pequeños hoteles y actividades anexas al turismo;

- fortalecimiento de los apicultores y agricultores para vender al turismo;

- certificación de los guías turísticos;

- crear medios gráficos de ayuda al turista que llega a la región. Folletos, libros técnicos sobre el parque, etc. 
v - Plano de hospitalidad regional y estético paisajístico

- Crear lugares de ocio para el poblador local y que también sirva al turista;

- Plano de urbanismo de accesibilidad y movilidad. Capacidad de acogida máxima. Frecuencias y flujos de turistas;

- Plano de salud, seguridad e informaciones regional.

\section{Consideraciones finales}

Las primeras consideraciones salen de la armonización de dos realidades regionales no siempre excluyentes:

- precariedad política, socio-institucional y económica de la región para desarrollar la actividad turística en el actual proceso competitivo de la globalización;

- el parque, con sus figuras prehistóricas, posee un alto volumen de potencial turístico en nivel internacional.

El proyecto de desarrollo sostenible, a partir del turismo como actividad transversal-integradora, debe ser el encargado en la armonización de esas dos realidades, objetivizando siempre el beneficio de la mayoría de la población local. Para eso, el proyecto debe conceptualizar que la mera construcción del aeropuerto y del complejo hotelero seria un crecimiento económico, y como afirma Rodríguez (2004), el crecimiento económico se refiere al aumento relativo del Producto Interior Bruto - PIB, en consecuencia, el crecimiento económico solo es un elemento más del desarrollo sostenible.

Para evitar que el turismo produzca grandes desequilibrios económicos, ambientales, culturales y sociales, se debe tener en cuenta (ver Figura 1):

- Las verticalidades. En este caso, las verticalidades son la alta inversión económica del grupo hotelero y del aeropuerto y el volumen de turistas que son flujos de la globalización que puede actuar como energía entrópica, sino existe una debida planificación preventiva regional.

- Las horizontalidades. Según los resultados de la investigación de Rodríguez (2004) los procesos culturales, políticos y sociales son los últimos en efectivizarse en la implementación de un proyecto en la actual globalización. Por eso hay que elaborar y gestionar programas que fortalezcan los flujos regionales de cohesión social, cooperación, capacitación, educación, solidaridad, que aumenten la calidad de vida, etc.

Por lo tanto, en la región de la Sierra de la Capibara es prioritaria la actuación preventiva del proyecto de desarrollo, o sea, antes de la entrada de las inversiones económicas se deben implementar programas en las esferas social, cultural y política. Esa acción preventiva podrá optimizar la convergencia de las verticalidades y horizontalidades que el turismo de masa generará en la región. Sino en las condiciones actuales de la región, la verticalidad de las inversiones tiene alta posibilidad de actuar como una energía entrópica regional y no dar tiempo a las horizontalidades formar un tejido sólido, capacitado y de calidad para ese tipo de actividad turística de masa.

Por otra parte, se constató la falta de implicación y participación de la población local, por lo tanto, aumenta la complejidad de la implementación de un proyecto de desarrollo sostenible, que de por si es dificultosa por las características de la región. Como afirma Molina (2005), en la región de la Sierra de la Capibara debe tenerse en cuenta un pacto político-social y empresarial, lo más amplio posible, que exija voluntad política-empresarial pero, también la implicación social regional, para poder equilibrar funcionalmente las diferentes etapas de los procesos sociales, culturales, políticos y económicos. O sea en los programas preventivos deben actuar proactivamente tanto la esfera pública, inversores internacionales y ONGs como también los agentes regionales y en especial la población, que debe actuar proactivamente para mejorar y enriquecer los procesos regionales.

A modo de conclusión, el turismo debe ser planificado preventivamente y transversalmente de manera que revitalice las demás actividades socio-económicas regionales y dé oportunidad a las nuevas actividades y sobre todo que aumente la calidad y solidez de los tejidos sociocultural, microeconómico y político de la región. Por lo tanto aunque actualmente no existe una configuración favorable para el turismo de masa, si puede existir la posibilidad de realizar un proyecto de desarrollo sostenible gracias al turismo internacional. El proyecto debe tener un perfil preventivo y mejorar la región, para la población local en primero lugar, para posteriormente, valorizar los ricos recursos turísticos regionales. Eso debe ser bajo el liderazgo técnico-institucional del turismo, como actividad transversal-coordinadora, que pueda integrar de manera sostenible las necesidades regionales y de los inversores y turistas internacionales. Así, el proyecto de desarrollo sostenible, mediante el turismo, debe esforzarse en buscar la sinergia de las horizontalidades y verticalidades, y sobretodo, preparar y configurar adecuadamente la población local y su región para ser seleccionados por los beneficios del actual proceso de globalización. 


\section{Referencias bibliográficas}

CAMAGNI, R. 1998. Beyond complexity un urban develoment studies. In BERGULIA et al. (Coord.). The city and its sciences. Heidelberg: Physica-Verlag.

2003. Incertidumbre, capital social y desarrollo local: enseñanzas para una gobernabilidad sostenible del territorio. Investigaciones Regionales, v. 2, p 31-57.

EMBRATUR. 2003. Turismo, estruturação e legislação. Rio de Janeiro: EMBRATUR.

FUNDHAM. 2003. Relatório anual.Piauí: FUNDHAM.

MÉNDEZ, R. 2003. Innovación y redes locales como estrategias de desarrollo territorial. Revista Catalana de Geografía, v. 76, p 177-198.

MOLINA, M. 2005. El desarrollo local: problemática y estrategias futuras. Rev. Zaragoza, p 37-50.

OMT. 2005. Guide for local authorities on developing sustainable tourism. London: OMT.

PIEMTUR. 2003. Dados estatísticos do turismo. Piauí: PIEMTUR.

PNUD. 2004. Informe sobre desarrollo humano 2003. Madrid: Mundi-Prensa.

RODRIGUEZ, A. 2004. Reestructuración socioeconómica y desequilibrio regionales en la Unión Europea. Madrid: Instituto de Estudios Económicos.

SANTOS, M. 2000. Por uma outra globalização: do pensamento único à consciência universal. Rio de Janeiro: Record.

1997. Espaço e método. São Paulo: Nobel.

1996. A natureza do espaço. Técnica e tempo. São Paulo: Huitec.

TOLEDO, G. et al. 2004. Tourism management in the context of regional strategic planning. INTERNATIONAL CONFERENCE ON MANAGEMENTE OF TECHNOLOGY - IAMOT, 13, Washington, DC.

TROITIÑO, et al. 2004. La red complementaria del sistema urbano español: un nuevo marco interpretativo. CONGRESO INTERNACIONAL DE ORDENACION DEL TERRITORIO, 3, Anais..., p 357-375.

TROITIÑO, M. 2003. Turismo y conjuntos monumentales: desafíos de interpretación y gestión. In: CONGRESO INTERNACIONAL SOBRE EL DESARROLLO TURÍSTICO INTEGRAL DE CIUDADES MONUMENTALES, Granada: PPTG., p 273-290.

1998. Turismo y desarrollo sostenible en las ciudades históricas. ERIA, n. 47, p. 211-227.

UCM. 2004. Turismo, ciudades históricas y estrategias de desarrollo. Madrid: Departamento de Geografía Humana, Universidad Complutense de Madrid.

UNESCO. 2004. Anuario mundial 1992. Madrid: unESCO.

VÁZQUEZ BARQUERO, A. 2002. Desenvolvimento endógeno en tempos de globalização. Porto Alegre: Ed. UFRGS.

VELTZ, P. 2001. Mundialización, cuidades y territorios. Barcelona: Ariel.

Recibido en: 20/08/2004.

Aprobado en: 22/09/2005. 